\title{
A Proposal for a Model Psychopharmacology Curriculum for Psychiatric Residents
}

\author{
Ira D. Glick, M.D., David S. Janowsky, M.D., Carl Salzman, M.D.,
} and Richard I. Shader, M.D.

In the early 1980s, there was an explosion of new knowledge in psychopharmacology and in the neurosciences underlying the field. As a result, there was an urgent need for a curriculum for planning and teaching basic and clinical psychopharmacology in psychiatric residencies. There was also a general consensus in the field that:

- too few residency programs were providing adequate teaching time and content to psychopharmacology;

- there was great variance in the effectiveness of such teaching, even when there was appropriate time and content;

- there was a need to assure adequate, science-based knowledge and skills among psychiatric residents; and

- resources for designing such educational programs were not uniformly distributed nationally.

From the Department of Psychiatry, The New York Hospital-Cornell University Medical Center, New York, New York (IDG), the Department of Psychiatry, University of North Carolina Medical School, City, North Carolina (DSJ), the Department of Psychopharmacology, Massachusetts Mental Health Center, and the Department of Psychiatry, Harvard Medical School (CS), and the Departments of Psychiatry and Pharmacology, Tufts University School of Medicine and New England Medical Center, Boston, Massachusetts (RIS). These authors represent a subcommittee of the American College of Neuropsychopharmacology, Box 1823-Station B, Vanderbilt University, Nashville, Tennessee 37235 . The views of the authors do not reflect official ACNP policy.

Address correspondence to Ira D. Glick, M.D., Department of Psychiatry, Payne-Whitney Psychiatric Clinic, New York Hospital-Comell Medical Center, 525 East 68th Street, New York, NY 10021.

Reprinted in part from Glick ID, Janowsky DS, Salzman C, Shader RI (1984): A Model Psychopharmacology Curriculum for Psychiatric Residents. Nashville, Tennessee, The American College of Neuropsychopharmacology.

Received September 9, 1991; revised December 20, 1991; accepted January 16, 1992.
The American College of Neuropsychopharmacology (ACNP) was asked by the American Psychiatric Association, the American Association of Directors of Psychiatric Residency Training, and numerous teachers of psychopharmacology to develop a structured neuropsychopharmacology curriculum and teaching aids. Accordingly, we present an abbreviated version ${ }^{1}$ of that document (Glick et al. 1984) to familiarize readers with the ACNP model and to report the findings of a small nonevaluation pilot test to determine its usefulness and problems. We emphasize that the present proposal is only a guide; diversity of curricula will very likely reflect the diversity of different departments, as it should.

\section{SUMMARY OF THE MODEL CURRICULUM}

A prior monograph (Glick et al. 1984) and paper (Meltzer 1987) have described the curriculum in detail. Here we provide an outline of the curriculum (Table 1) and its essence. It has been shortened largely through the omission of examples and the lecture outlines.

\section{OVERVIEW}

This teaching package assumes that there is not only psychopharmacologic "theory and practice" to be taught, but also underlying principles to be learned. Psychiatric residents learn in different ways, at different speeds, and in very different settings, and repetition of appro-

\footnotetext{
${ }^{1}$ It is not our intent to republish the full content and lecture outlines here. Readers may obtain the full curriculum by contacting the Secretariat of the ACNP.
} 
Table 1. Outline of the Model Curriculum

Objectives
Overview
What and How To Teach
Didactic program
Development of individual or small group supervision
Development of psychopharmacology units
Use of psychopharmacology-psychobiology journals
and newsletters
Representative basic psychopharmacology books
Development of psychiatric neurobiology lecture series
How to Evalute
Formal examination
Charting patterns
Supervisor's evaluation
Trainee evaluation of program
How to Integrate Psychopharmacology into an Ongoing
Residency Curriculum ("The Politics")
National level
Local level
Clinical Psychopharmacology: Relationship of Research
to Teaching
Psychopharmacology Curricula: The Past and the Future
Appendices

priate concepts and data at various steps in the residency is necessary for the integration and consolidation of this information base. Case-based learning is essential. The involvement of senior supervisors, who can model the integration of psychopharmacology into the total treatment planning for care, is also important.

We believe that the terminal objectives of a clinical psychopharmacology program should be to impart the ability to

- integrate psychodynamic-psychosocial and psychobiologic-psychopharmacologic aspects of a given patient's care;

- use psychotropic drugs safely;

- know when and when not to use psychotropic drugs;

- understand the limitations of psychotropic usage, their dangers, and pitfalls;

- possess reasonable theoretical models to understand the biologic mechanisms underlying the use of psychotropic drugs.

We have not included enabling objectives (i.e., what should be learned in each year), because they are too dependent on local program conditions (e.g., whether residents start psychiatry on inpatient or outpatient rotations or in emergency room settings, and the sequencing of other curricula). We have also not included material on the legal, regulatory, and ethical aspects of psychopharmacologic prescribing practices. For example, programs should include material on the rights of physicians in emergency clinical situations (e.g., when treating suicidal or assaultive patients) and the rights of patients to refuse treatment.

\section{WHAT AND WHO TO TEACH}

Obviously, each program must develop its own style for teaching psychopharmacology based on its resources, expertise, and available clinicalarenas. The following are suggestions for developing an optimal teaching curriculum and program. We have not delineated "priorities versus the ideal.": Therefore, although each program will have to decide that question, we emphasize that didactic lectures and the journal club conference have been the "irreducible minimum" as opposed to the fullest program possible in the best of all worlds.

The question of which learning groups should be interdisciplinary must be considered, since many beginning residents are ashamed to reveal their rudimentary knowledge in psychopharmacology in front of, for example, ward nurses and other personnel. A survey of the content and formats of what we consider the better programs that include psychopharmacology teaching is found in Appendix A of the full monograph (Glick et al. 1984).

\section{DIDACTIC PROGRAM}

\section{A Psychopharmacology Lecture Series}

Such a lecture series would cover the basic and sophisticated uses of psychotropic drugs, including development, advantages/disadvantages, and integration with other formats. We view the didactic curriculum as being taught at three different levels $-a$ crash course (taught in the PG I year or in the summer of the PG II year), a basic course, and an advanced course with components taught sequentially during the four years of training. In addition, formal didactic teaching of psychopharmacology should be provided within the large context of the teaching of neurobiology and biological psychiatry.

In the next two sections, we present a list of general issues and concepts as well as specific topics that may be helpful for developing a didactic lecture series. Suggested introductory themes are delineated. Both the issues and the topics are also appropriate for consideration in the more mentorial and supervisory forms of teaching psychopharmacology, such as in psychopharmacology case conferences or psychopharmacology rounds. In addition to serving as guides to the content of didactic lectures, such outlines may be helpful in preparing a series of slides for use in didactic lectures.

Appendix B in the full monograph (Glick et al. 1984) presents model lecture outlines, which were readily available to us and which may be useful in organizing 
a lecture series for residents. The lecture outlines are representative and are not "model" outlines in the sense of being without flaw. It is hoped that they offer useful guidelines for the preparation of similar outlines.

General issues and concepts. Table 2 lists suggested general issues and concepts worth covering in specific clinical psychopharmacology lectures about a given drug or class of drugs. The level of the course (i.e., basic or advanced) should determine which of these to include).

Specific topics. Table 3 lists a series of lecture topics of importance in constructing a didactic psychopharmacology series. We recognize that the list is long and the topics may not seem to be of parallel importance, but we leave the job of tailoring the topics to local program coordinators.

The first nine topics are most appropriate for the first-year resident who needs to rapidly master the use of psychotropic agents. Such lectures may occur within the context of a crash course offered in a resident's first year of psychiatry training. A crash course covers issues of diagnosis (i.e., DSM-III-R) and treatment with antipsychotics and antidepressants, emphasizing indications, contraindications, and dose regimens includ-

Table 2. General Issues and Concepts Worth Covering in Specific Clinical Psychopharmacology Lectures

- Proposed mechanisms of action of drug

- Basic pharmacologic issues (pharmacokinetics, physiologic effects, etc.)

- Diagnostic issues (overlap between efficacies, delineation of syndromes, etc.)

- Efficacy of drugs in related or complementary classes vs. each other and placebo

- Age-related issues (pediatric, geriatric, etc.)

- Drug dosages and timing

- Specific indications (specific use of drugs, differential efficacies among drugs)

- Uses of drugs in nonpsychiatric settings

- Uses across diagnoses (specificity issues)

- General principles of drug use (models of administration, timing of dosages, use of blood levels, predictors of effect, etc.)

- Drug-drug interactions (psychotropic-psychotropic, medical-psychotropic, recreational-psychotropic)

- Drug combination therapies

- Side effects (CNS, metabolic, cardiovascular, dermatologic, peripheral autonomic, electrocardiographic)

- Medical, laboratory workups needed for a given drug

- Drug withdrawal effects

- Oversdose signs, effects, treatment

- Addiction-habituation-dependency potential

- Contraindications

- Uses of blood levels

- Issues of acute-, continuation-, maintenance-phase psychopharmacology

- Strategies for treatment-resistant patient
Table 3. Possible Lecture Topics in Clinical

Psychopharmacology

- General principles

- Biologic hypotheses (genetic, biochemical, electrolyte, circadian)

- Antipsychotic drugs

- Antidepressants

- Antimanic agents, mood normalizers

- Antianxiety agents

- Child psychopharmacology

- Liaison consultation psychopharmacology

- Geriatric psychopharmacology

- Treatment of resistant depression and mania

- Treatment of resistant schizophrenia

- Treatment of resistant anxiety, panic disorder, agoraphobia

- Exotic uses of psychotropic drugs

- Drug treatment of sleep disorders

- Precursor loading therapies in schizophrenia and depression

- Substance abuse, recreational drug use

- Alcohol

- Drug treatment of personality disorders

- Interface of psychopharmacology with other modalities (psychotherapy, electroconvulsive therapy, etc.)

- Psychotropic drug-drug interactions

- "Sociopsychology" of psychopharmacology

- When to stop, withhold, or not start drugs (issues of why to use drugs, change doses, continue medications)

- Sociology of giving medicine (influence of social status, age, site of treatment on drug treatment)

- Patient education (drug effects, compliance, side effects)

- Patient resistance to/overuse of drugs

- Roles, settings, and sociology of psychotropic drug use and abuse

- Electroconvulsive therapy

- Research approaches in psychopharmacology (instruments, design, etc.)

ing routes of administration and side effects. It may be useful to repeat such lectures in greater depth in the second half of the PG II year, after the beginning resident has had more integrated clinical experience. In general, Table 3 lists these topics in order of how they could be presented over the course of the residency.

\section{Other Didactic Elements}

Literature review seminar (journal club) and outside guest lecturer series. We also recommend an integrated literature review seminar (or journal club) and guest lecturer series for all three years of residency training in which the literature read relates directly to the lecture presented.

Formal case conference. Case conferences should be offered in all three years of psychiatric residency training. Patients are selected because of difficulties in their treatment, particular features in their clinical presenta- 
tions, or because they illustrate a particular aspect of psychopharmacology.

Continuous clinical psychopharmacology case conferences focusing on the integration of psychotherapy and psychopharmacology. Another didactic technique is the use of a continuous longitudinal clinical psychopharmacology case conference, occurring approximately twice monthly, illustrating the need for psychopharmacologic interventions and other psychotherapies.

Individual or small group supervision. Supervision may involve use of small group, clinical, mentorial teaching by psychopharmacology experts using selected case material. Modeled after individual or small group psychotherapy supervision, the discussion may focus on the effects of role and setting on the trainee or on the tendency to treat patients entirely by diagnosis, socioeconomic status, and location of treatment.

ECT can also be taught as part of the psychopharmacology curriculum. The most effective form of teaching is during the actual administration of ECT, emphasizing leaming through practice and observation.

Supervision of junior residents in situ in such settings as drug clinics, inpatient units, and emergency rooms may also be provided by senior residents (i.e., PGY IIIs and IVs).

Development of psychopharmacology units. It may be helpful to develop specific psychopharmacology treatment units as subcomponents of outpatient psychiatry rotations and/or liaison-consultation units.

Reading lists. Introduction to the relevant psychopharmacologic literature is important in the training of residents in psychopharmacology. Appendix $\mathrm{C}$ in the full monograph (Glick et al. 1984) gives references that may be helpful.

Psychopharmacology-psychobiology journals and newsletters. A complete annotated list of journals, newsletters, and texts is given in the full monograph (Glick et al. 1984).

Psychiatric neurobiology lecture series. In some programs, it may be useful to provide a series of lectures and didactic seminars on the most up-to-date thinking on the current neurobiologic concepts underlying psychiatry and psychopharmacology.

\section{HOW TO EVALUATE}

Both to understand whether a given clinical psychopharmacology program is achieving its teaching goals and to point out areas of weakness in individual trainees, several standardized techniques are available to evaluate trainee competence before and after curriculum exposure.

Optimal evaluation of a clinical psychopharmacology program should consist of preeducation and post- education formal examinations, reviews of the trainees' charting patterns, and ongoing written evaluation by psychopharmacology supervisors. Resident knowledge and skills may also be evaluated during a "mock boards" type of clinical examination at least three times during the residency.

\section{RESULTS OF THE FOLLOWUP EVALUATIONS}

Because we believed that a follow-up evaluation of the model curriculum was crucial for its improvement, and because initially there was a very positive anecdotal but nonsystematic response to its publication and use, we conducted two pilot surveys. The first was performed one year after distribution in 1986, and the second, after four years, in 1989. Both surveys used closed- and open-ended questions focusing on usefulness, including (in the latter) a question on which elements were most or least useful. The questionnaire was sent to all directors of psychiatric residency education in the United States (160 in 1989); it asked them or their designated principal psychopharmacology teacher to respond. As an attempt to comprehensively study the effect of the curriculum, the surveys were unsuccessful because of poor response rate, so the only findings that can be shared are the most preliminary of impressions.

The second survey, which used a more structured questionnaire than the first, elicited 67 responses. Of these, 33 respondents said they had received the curriculum, while the remaining 34 requested a copy, suggesting that it had not been passed on from the prior training director or coordinator of psychopharmacology teaching (or that it had not been received in 1985). Of the 33 who had received it, 21 said they were using it; of these, the mean rating of usefulness was " 4 " on a 5-point scale, with " 5 " designating "very useful," and " 0 " designating "not useful at all." The scale was undirectional - there were no levels for negative ratings.

Based on 21 responses, the rank order of the components reported as most useful were, in descending order: the content describing what to teach (mean rating, 4.3); the lecture outlines (mean rating, 4.0); the teaching methods (mean rating, 3.4); the evaluation methods (mean rating, 3.0); and the section on the "politics" of psychopharmacology teaching (mean rating, 2.3). What seemed to be most popular, based on anecdotal reports, were the lecture outlines. Parenthetically, a major problem was explaining to the users that these were models of possible lectures rather than a "textbook of psychopharmacology." Most of the respondents found all of the components useful.

Alternatively, the 12 respondents not using the curriculum identified four major reasons for not using it. Eleven of the 12 indicated that they were already using 
most of the suggestions, 9 said they had no one (besides themselves) to teach it all, 9 had insufficient curriculum time to teach it all, and 8 found it impractical to implement all of it in their settings.

Finally, all 21 of those who did use the curriculum reported that they believed it improved both the teaching and the learning of psychopharmacology at their institutions.

The final problem - and a potentially remediable one-was the fact that many individuals who received the model curriculum did not pass it on when their responsibilities shifted. A related issue concerns the nature of the field (compared with other areas in psychiatry). Psychopharmacology is rapidly changing as we develop new medications and treatment strategies and as continued advances occur in the neurosciences and other basic sciences of psychopharmacology. Accordingly, what is required by many residency programs (but difficult to find) is a teacher-clinicianresearcher who keeps up with the latest advances in the field, i.e., a psychopharmacology teacher who can "jump on and off a rapidly moving train," who can keep up with the literature and translate the new information into patient care-giving principles to the students who are the life blood of the field.

\section{CONCLUSION}

In conclusion, the data suggest that for some programs a systematically designed model curriculum is useful in the teaching of psychopharmacology. Furthermore, it seems desirable for the mechanisms to be in place to upgrade model curricula every two to three years.

\section{ACKNOWLEDGMENTS}

This work was supported in part by National Institute of Mental Health grant 83MO5598951OD.

\section{REFERENCES}

Glick ID, Janowsky DS, Salzman C, Shader RI (1984): A Model Psychopharmacology Curriculum for Psychiatric Residents. Nashville, Tennessee, The American College of Neuropsychopharmacology

Meltzer HY (ed), Psychopharmacology: The Third Generation of Progress. New York, Raven Press, (1987) pp 13131321 